\title{
Les jeunes chercheuses sous la direction d'un directeur de recherche homme : perception de leur relation d'accompagnement
}

Females enrolled in master degree programs supervised by male supervisors: a perception of their supervision

Las investigadoras en formación bajo la dirección de un investigador de sexo masculino: percepción de la relación de acompañamiento

\section{Laëtitia Gerard}

Volume 43, numéro 1, 2017

Texte reçu le : 19 mars 2016

Version finale reçue le : 2 mai 2017

Accepté le : 17 mai 2017

URI : https://id.erudit.org/iderudit/1042076ar

DOI : https://doi.org/10.7202/1042076ar

Aller au sommaire du numéro

Éditeur(s)

Revue des sciences de l'éducation

ISSN

1705-0065 (numérique)

Découvrir la revue

Citer cet article

Gerard, L. (2017). Les jeunes chercheuses sous la direction d'un directeur de recherche homme : perception de leur relation d'accompagnement. Revue des sciences de l'éducation, 43(1), 116-140. https://doi.org/10.7202/1042076ar
Résumé de l'article

Notre recherche est partie de deux constats : 1) à l'entrée en master, l'étudiant expérimente pour la première fois une nouvelle relation pédagogique, duale, à savoir la relation d'accompagnement de mémoire; 2) en master, les jeunes chercheuses sont plus souvent accompagnées dans leur recherche par un directeur du sexe opposé que les étudiants. Comment les jeunes chercheuses accompagnées dans leur recherche par un directeur - homme perçoivent-elles cette relation pédagogique à deux? Quelles perceptions ont-elles d'elles-mêmes et de leur directeur de recherches, et ce, au niveau des trois dimensions : scientifique, institutionnelle et relationnelle? Pour mener cette recherche, nous avons interrogé onze jeunes chercheuses inscrites en master 2. Les résultats montrent que les jeunes chercheuses perçoivent leur rôle, le rôle de leur directeur et leur relation d'accompagnement de manière très scolaire et hiérarchisée. Elles éprouvent par ailleurs des difficultés à communiquer avec leur directeur de recherches (dimension institutionnelle) et à gérer la relation interpersonnelle (dimension relationnelle). Enfin, la dimension relationnelle est considérée par les jeunes chercheuses comme le point central de la relation d'accompagnement et constitue leur principale source d'insatisfaction.
Tous droits réservés @ Revue des sciences de l'éducation, 2017
Ce document est protégé par la loi sur le droit d'auteur. L'utilisation des services d'Érudit (y compris la reproduction) est assujettie à sa politique d'utilisation que vous pouvez consulter en ligne.

https://apropos.erudit.org/fr/usagers/politique-dutilisation/ 


\section{Les jeunes chercheuses sous la direction d'un directeur de recherche homme : perception de leur relation d'accompagnement ${ }^{1}$}

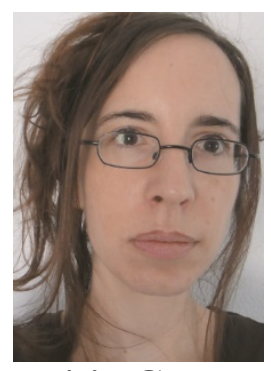

Laëtitia Gerard

Docteure en sciences de l'éducation

Université Paris 13

RÉSUMÉ - Notre recherche est partie de deux constats : 1) à l'entrée en master, l'étudiant expérimente pour la première fois une nouvelle relation pédagogique, duale, à savoir la relation d'accompagnement de mémoire; 2) en master, les jeunes chercheuses sont plus souvent accompagnées dans leur recherche par un directeur du sexe opposé que les étudiants. Comment les jeunes chercheuses accompagnées dans leur recherche par un directeur - homme perçoivent-elles cette relation pédagogique à deux? Quelles perceptions ont-elles d'elles-mêmes et de leur directeur de recherches, et ce, au niveau des trois dimensions : scientifique, institutionnelle et relationnelle? Pour mener cette recherche, nous avons interrogé onze jeunes chercheuses inscrites en master 2. Les résultats montrent que les jeunes chercheuses perçoivent leur rôle, le rôle de leur directeur et leur relation d'accompagnement de manière très scolaire et hiérarchisée. Elles éprouvent par ailleurs des difficultés à communiquer avec leur directeur de recherches (dimension institutionnelle) et à gérer la relation interpersonnelle (dimension relationnelle). Enfin, la dimension relationnelle est considérée par les jeunes chercheuses comme le point central de la relation d'accompagnement et constitue leur principale source d'insatisfaction.

MotS CLÉS - Supervision, direction de recherches, accompagnement, sexe, étudiante.

\section{Introduction et problématique}

Dans cet article, nous allons nous intéresser au diplôme de master. Le système universitaire français est composé de trois cycles. Le premier cycle, la licence, se déroule sur trois années. Le deuxième cycle, le master, se déroule sur deux années. Le doctorat est le troisième cycle avec une durée officielle de trois ans. La première année de master est nommée le master 1, et la deuxième année, le master 2. La formation de master comporte des cours et une initiation à la recherche, avec notamment la rédaction d'un mémoire (arrêté du 22 janvier 2014).

\footnotetext{
${ }^{1}$ Nous tenons à remercier tous les participants à cette étude.
} 
La rédaction d'un mémoire implique une relation pédagogique nouvelle, à savoir une relation d'accompagnement directeur de recherches - jeune chercheur. On observe donc une évolution de la relation pédagogique enseignant-enseigné de la licence au master. En licence, la relation de l'étudiant avec son enseignant est groupale, c'est-à-dire que l'étudiant se situe au sein d'un groupe-classe, face à un individu enseignant. Cette relation groupale tend à dissimuler l'étudiant dans et par le groupe, et à l'anonymiser auprès de l'enseignant (Langevin et Bruneau, 2000). Les auteures insistent sur le lien purement professionnel qui lie l'étudiant et l'enseignant en licence. À l'entrée en master, comme le soulignent Le Bouëdec et de La Garanderie (1993), la structure formative évolue. L'étudiant expérimente pour la première fois la relation pédagogique duale d'accompagnement, qui n'est pas toujours simple à appréhender :

Beaucoup d'étudiants très à l'aise jusque-là [en Licence] dans les enseignements dont ils tirent un éclairage pour eux important, se heurtent maintenant [en master] à une logique de formation assez radicalement différente, qui les déroute beaucoup. Le nombre de mémoires ou de thèses jamais achevés, le gâchis social et universitaire qui en découle, seraient, à eux seuls, des raisons largement suffisantes pour s'interroger sur l'accompagnement et le suivi des études doctorales d'une part, et sur les stratégies de formation àlet par la recherche par l'autoformation d'autre part (Le Bouëdec et de La Garanderie, 1993, p.12).

Pour réussir, l'étudiant doit donc développer des compétences et des stratégies nouvelles par rapport à celles qu'il a pu expérimenter en licence. Il doit notamment se familiariser avec son nouveau rôle d'apprenti-chercheur, mais aussi avec le nouveau rôle d'accompagnateur de l'enseignant. Il doit apprendre à adopter un comportement verbal et non verbal approprié, un nouveau mode de communication. Il lui faut aussi apprendre à gérer la relation interpersonnelle professionnalisante et la part d'affect, qui est plus importante dans la relation pédagogique duale que dans une relation pédagogique groupale.

En France, la probabilité qu'une jeune chercheuse inscrite en master soit accompagnée dans sa recherche par un directeur de sexe masculin est plus forte que celle d'être accompagnée par une directrice. En effet, pour l'année 2013-2014, il y avait 59,2 \% de jeunes chercheuses inscrites en master. Elles sont notamment majoritaires en lettres, langues et sciences humaines (73,1\%), en droit, sciences politiques (64,7\%) et en économie avec 55,2 \% (Karpenko et Gidycz, 2012). Parmi les directeurs de recherche, en 2013, 56,8\% des maitres de conférences et $78 \%$ des professeurs sont des hommes. Ainsi, en master, comme en doctorat, les binômes jeune chercheuse-directeur de recherche homme restent les plus fréquents. 
Conséquemment, nous formulons deux constats : 1) À l'entrée en master, l'étudiant expérimente pour la première fois une relation pédagogique duale: la relation d'accompagnement; 2) en master, les jeunes chercheuses sont plus souvent accompagnées dans leur recherche par un directeur du sexe opposé que les jeunes chercheurs. Notre question de recherche est la suivante : Comment les jeunes chercheuses accompagnées dans leur recherche par un directeur - homme perçoivent-elles cette relation pédagogique à deux? Quelles perceptions ont-elles d'elles-mêmes et de leur directeur de recherches? S'intéresser aux jeunes chercheuses constitue un enjeu majeur au regard des écrits de recherche trop peu nombreux sur le sujet, mais qui pointent déjà d'énormes dysfonctionnements au niveau de la relation avec leur directeur de recherche. Nous les détaillerons dans la partie théorique; il s'agit notamment de problèmes communicationnels, relationnels, et de différences de traitement par rapport à une relation d'accompagnement avec un jeune chercheur.

\section{Contexte théorique}

Nous parlerons ici de jeune chercheuse pour désigner à la fois les jeunes chercheuses inscrites en master et les doctorantes.

\subsection{Les trois dimensions de l'accompagnement de recherches}

D'un point de vue sémantique, accompagner, c'est se joindre à quelqu'un pour aller où il va en même temps que lui (Paul, 2004, p. 308). En s'appuyant sur cette définition de l'accompagnement, nous définissons l'accompagnement de recherches comme une relation pédagogique duale impliquant un novice et un expert dans un cheminement commun, mais transitoire, vers le développement de compétences de recherche. Le cheminement est commun dans le sens où le directeur de recherches est une personne ressource qui apporte son aide au jeune chercheur dans sa formation à la recherche, pour l'accompagner dans sa recherche et la rédaction de son mémoire. L'accompagnement est également transitoire : le directeur s'intéresse à l'individu en devenir et lui apporte son aide dans sa formation par la recherche pour lui permettre de développer des compétences de chercheur, et cette aide prend fin à l'obtention du diplôme.

Nous envisageons l'accompagnement de recherches autour de trois dimensions: scientifique, institutionnelle et relationnelle (Gerard, 2009, 2014) : 
- la dimension scientifique : l'étudiant qui entre en master devient un apprenti-chercheur qui se forme à la recherche par la recherche, accompagné par un chercheur expérimenté (Germain et Gremillet, 2000; Leduc, 1990). Cette dimension inclut ce qui a trait à la construction du travail de recherche de l'étudiant, pour lequel il est amené à se situer en tant qu'apprenti-chercheur dans une formation à la recherche par la recherche. Elle concerne plus précisément les aides théoriques, méthodologiques et rédactionnelles apportées par le directeur de recherches;

- la dimension institutionnelle : à l'entrée en master, on observe une évolution de la part d'autodirection (Carré, 2005; Knowles, 1975) de l'étudiant. Cette dimension correspond à l'organisation institutionnelle de l'accompagnement et à la prise en charge, par le jeune chercheur, de l'organisation de sa formation, avec une accentuation de sa part d'autodirection. Cette dimension traite notamment des modalités d'entrée de l'étudiant en master, des modalités de constitution du binôme directeur-jeune chercheur, et des modalités de communication et de rencontre de ce binôme. Elle recouvre aussi la construction de la planification du travail et de la recherche, ainsi que les différents aspects liés à la temporalité du travail de recherche, tels que les délais pour la remise d'écrits et pour les rétroactions, ainsi que la fréquence et la durée des rendez-vous;

- la dimension relationnelle : à l'entrée en master, l'étudiant n'est plus anonymisé dans et par le groupe; il découvre une nouvelle relation à l'enseignant: la relation pédagogique enseignant-enseigné devient duale. Cette dimension correspond à la relation interpersonnelle qui se construit entre le directeur de recherches et le jeune chercheur. Cette troisième dimension concerne notamment le type de soutien attendu par le jeune chercheur et envisagé par le directeur de recherches, et les types de discussions qui s'instaurent au sein du binôme. Elle concerne aussi les formes de rétroaction utilisées par le directeur, par exemple la tonalité évaluative, et certains marqueurs verbaux comme le tutoiement/vouvoiement.

Notre recherche vise à étudier comment les jeunes chercheuses a) accompagnées dans leur recherche par un directeur - homme, et b) qui expérimentent pour la première fois une relation pédagogique duale, se perçoivent, perçoivent leur directeur-accompagnateur et leur relation d'accompagnement, et ce, au niveau des trois dimensions: scientifique, institutionnelle, relationnelle. Nous allons commencer par nous intéresser aux recherches déjà menées sur ces binômes mixtes. 


\section{2 État de la recherche sur les binômes mixtes directeur de recherches-jeune chercheuse}

Peu de recherches se sont intéressées spécifiquement à ces binômes mixtes. Nous allons en citer ici une vingtaine; majoritairement anglophones, parfois anciennes, elles se concluent toutes sur des résultats plutôt négatifs concernant le fonctionnement des binômes directeur homme - jeune chercheuse. Notons que les résultats de recherches francophones et anglophones menées sur l'accompagnement de mémoires et de thèses varient peu en fonction des pays.

\subsubsection{Degré de satisfaction}

Premièrement, les recherches montrent que les jeunes chercheuses accompagnées dans leur recherche par des directeurs sont moins satisfaites de leur direction de recherches que les jeunes chercheurs et jeunes chercheuses appartenant à d'autres binômes (Conrad, 1994; Gillespie, Walsh, Winefield, Dua, et Stough, 2001; Seagram, Gould, et Pyke, 1998). Cette insatisfaction pourrait s'expliquer par une vision différente de la relation d'accompagnement. Les recherches (Hammick et Acker, 1998; Juniper, Walsh, Richardson, et Morley, 2012) montrent en effet une différence de perception relative à l'accompagnement et au rôle de chacun. Les jeunes chercheuses sont dans l'attente d'une certaine proximité relationnelle, c'est-à-dire proche d'une relation amicale, alors que les directeurs sont davantage dans l'attente d'une relation scientifique; ils séparent la jeune chercheuse de la personne, la relation professionnelle est uniquement centrée sur la tâche à accomplir.

\subsubsection{Difficultés de socialisation au métier de chercheur}

Les attentes des jeunes chercheuses seraient plutôt en adéquation avec l'approche des directrices de recherches, parce qu'elles considèrent qu'il est important de connaître aussi la jeune chercheuse en tant qu'individu, étant donné que les éléments de la vie personnelle peuvent influer sur la qualité du travail (Gerard, 2009). Au sein du binôme jeune chercheuse - directeur, cette différence de perception relative à la relation d'accompagnement peut avoir des effets négatifs sur la réussite de la jeune chercheuse si elle n'est pas discutée au sein du binôme (Gerard, 2009; Herzlich, 2002). En l'absence de toute explicitation du rôle de chacun, la jeune chercheuse pourrait interpréter la distance relationnelle instaurée par son directeur comme de l'exclusion, une négation de sa personne, ce qui pourrait entraîner un retrait partiel (absence de contact) préjudiciable au développement de ses compétences de chercheuse, ou définitif (son abandon). 
L'insatisfaction des jeunes chercheuses relative à leur relation d'accompagnement pourrait s'expliquer également par la prédominance de préjugés machistes ressentis. Boyd et Wylie (1994) montrent que les jeunes chercheuses ont la sensation de ne pas être prises au sérieux et de devoir en faire davantage que leurs homologues masculins pour être prises au sérieux (Lafay, Manzanera, Papet, Marcelli, et Senon, 2003). Cette sensation peut avoir un effet négatif sur leur sentiment d'auto-efficacité (Bandura, 2003) et, de ce fait, sur leur réussite et le développement de leurs compétences.

2) Deuxièmement, plusieurs recherches ont montré que la relation duale directeur homme jeune chercheuse pouvait avoir un effet négatif sur la socialisation de la jeune chercheuse. Par exemple, Over, Over, Meuwissen et Lancaster (1990) se sont intéressés au nombre de publications des doctorants en fonction du sexe de leur directeur. Leur recherche montre que le sexe du directeur n'a pas d'effet sur le nombre de publications. Par contre, les auteurs mentionnent qu'être accompagné par un directeur du même sexe peut constituer un avantage d'une autre manière : dans la carrière et dans le développement des compétences académiques, tel qu'enseigner, faire de la recherche ou écrire. La socialisation de la jeune chercheuse au métier de chercheur se retrouve également mise à mal lorsque les rencontres avec son directeur sont peu fréquentes. Schmidt et Umans (2014) montrent que les jeunes chercheuses passent moins de temps avec leur directeur, et le contactent moins fréquemment que leurs homologues masculins (Powles, 1989). Elles se privent ainsi d'une aide essentielle à l'avancement de leur travail de recherche et au développement de leurs compétences.

De la même manière, les moments informels entre le directeur et la jeune chercheuse seraient moins fréquents que dans les autres binômes. Phillips et Pugh (1994) remarquent en effet que les jeunes chercheuses ne sont pas toujours incluses dans ces moments de socialisation informels, tels que les apéritifs organisés par le laboratoire. Les auteurs émettent ainsi deux hypothèses. La première hypothèse concerne l'autosélection : les jeunes chercheuses éviteraient les rencontres informelles parce qu'elles ne se sentiraient pas à l'aise dans ce genre de situation ou parce qu'elles privilégieraient leurs obligations familiales. La deuxième hypothèse a trait aux directeurs de recherche : ils n'inviteraient pas les jeunes chercheuses parce qu'ils ne se sentiraient pas à l'aise et ne sauraient pas comment communiquer avec elles en tant qu'égaux. Dans les deux cas, la situation est dommageable pour la socialisation et l'insertion professionnelle de la jeune Le Bouëdec et De La Garanderie (1993) du réseau. Phillips et Pugh (1994) pointent d'autres 
préjugés des directeurs à l'égard des jeunes chercheuses : ils limiteraient leur critique dans le but d'éviter une réaction émotionnelle de la part de celles-ci. Les retours constructifs sont donc moins nombreux, ce qui aurait un effet sur l'avancement et la qualité de leur recherche. D'autres recherches abordent les préjugés, mais sous l'angle du regard des autres. Lee et Boud (2009) souligne qu'une relation de proximité relationnelle entre le directeur et la jeune chercheuse peut être interprétée par les autres membres du laboratoire comme une attirance sexuelle. De ce fait, nous pouvons postuler que certains directeurs se protègent du regard des autres en maintenant une distance relationnelle excessive, strictement professionnelle, qui pourrait être préjudiciable à la socialisation de la jeune chercheuse.

\subsubsection{Dépendance propice au harcèlement}

Troisièmement, la plupart des recherches qui ont été menées sur les binômes directeur homme jeune chercheuse portent sur le harcèlement moral ou sexuel. Phillips et Pugh (1994) évoquent, par exemple, l'abus de pouvoir qui peut conduire au harcèlement sexuel ou à l'exploitation. Certains directeurs n'hésitent pas à imposer leur domination masculine par de l'intimidation et de l'humiliation (Conrad, 1994), et à imposer leur pouvoir institutionnel en considérant les jeunes chercheuses qu'ils accompagnent comme des objets sexuels (Heinrich, 1991; Herzlich, 2002; Royer, 1998). Les jeunes chercheuses se situent dans une relation de dépendance vis-à-vis de leur directeur, relation qui s'avère particulièrement propice au harcèlement sexuel. Refuser les avances de celui-ci ou en faire part à un tiers n'est pas simple lorsque la jeune chercheuse a besoin de son accord et de lettres de recommandation pour de nombreuses activités et démarches (Collectif de lutte antisexiste contre le harcèlement sexuel dans l'enseignement supérieur, 2014; Dahan, 2007). Elle peut craindre que sa non-coopération ait une incidence directe sur sa recherche et constitue un frein à son insertion professionnelle.

En résumé, d'après les résultats de ces recherches, les binômes directeur-jeune chercheuse rencontreraient des difficultés au niveau des trois dimensions :

- Dimension scientifique : sentiment de ne pas être prise au sérieux;

- Dimension institutionnelle : difficulté de communication (contacts moins fréquents);

- Dimension relationnelle: insatisfaction concernant la relation interpersonnelle, distance relationnelle du directeur (mal à l'aise, peur du regard des autres), préjugés machistes (elles ne 
sont pas à la hauteur, elles pleurent quand on critique leur travail), harcèlement sexuel.

Les difficultés rencontrées au niveau des trois dimensions ont des effets négatifs sur la formation à la recherche par la recherche de la jeune chercheuse, à savoir le développement de ses compétences, sa socialisation et son insertion professionnelle.

Au vu des enjeux, il paraît essentiel de porter notre attention sur ces binômes à risque. Or, aucune des recherches précédemment citée n'a été spécifiquement menée sur ce type de binômes. Il s'agit principalement de recherches dont l'objet initial portait sur l'accompagnement de recherches, et dont les résultats ont fait apparaître, de manière sporadique, quelques faits saillants concernant ces binômes.

Pour cette raison, nous avons choisi de nous intéresser spécifiquement aux binômes directeurs de recherches homme-jeune chercheuse afin de recueillir les témoignages des jeunes chercheuses concernant leur perception d'elles-mêmes et de leur directeur dans le cadre de leur relation duale d'accompagnement.

\section{Méthodologie}

\subsection{Sujets}

Nous avons réalisé des entretiens semi-directifs auprès de 11 jeunes chercheuses inscrites en master 2 et accompagnées dans leur recherche par un directeur de sexe masculin. Parmi les 11 jeunes chercheuses, 9 sont en formation initiale, âgées de 23 à 25 ans, tandis que 2 sont en formation continue, âgées de plus de 45 ans; 6 jeunes chercheuses sont inscrites en sciences de l'éducation, 4 en sociologie et une en psychologie. Elles sont issues de 5 universités différentes, dans l'est, l'ouest et le nord de la France (voir Tableau 1). Nous avons choisi la France pour des raisons organisationnelles, mais aussi parce qu'aucune recherche française ne s'est encore intéressée aux binômes que nous avons choisi d'étudier. Concernant le choix de l'échantillon, il s'agit d'un choix par défaut. En effet, nous avons contacté 24 directeurs de laboratoires de recherche en psychologie, sociologie et sciences de l'éducation en France. Sur les 24 contactés, 7 ont accepté de nous fournir les adresses courriels de leurs étudiants inscrits en master 2. C'est ainsi que 156 étudiants ont ainsi été sollicités par courriel pour un entretien. Au total, nous avons obtenu seulement 27 accords d'entretiens de la part des étudiants, dont 11 jeunes chercheuses accompagnées dans leur recherche par un directeur homme. Nous avons choisi d'interroger, sur le terrain, l'ensemble de ce groupe d'étude. Ce faible nombre d'acceptations montre à quel point 
le sujet traité reste délicat. Les entretiens se sont déroulés en cours d'année et un suivi de leur réussite a été effectué 4 mois après la fin de l'année universitaire. Nous avons pu observer que sur les 11 jeunes chercheuses, 6 ont obtenu 16 sur 20 à leur mémoire de recherche, une a obtenu la note de 14, et 4 jeunes chercheuses ont choisi d'abandonner.

\section{Tableau 1}

Caractéristiques des jeunes chercheuses interrogées

\begin{tabular}{|c|c|c|c|c|c|}
\hline & $\begin{array}{c}\text { Formation } \\
\text { initiale ou } \\
\text { continue }\end{array}$ & Université & Discipline & $\begin{array}{l}\text { Note au } \\
\text { mémoire }\end{array}$ & $\begin{array}{c}\text { Poursuite en } \\
\text { doctorat }\end{array}$ \\
\hline $\begin{array}{l}\text { Jeune } \\
\text { chercheuse E1 }\end{array}$ & Initiale & U1 & $\begin{array}{c}\text { Sc.de } \\
\text { l'éducation }\end{array}$ & 16 & ne sait pas \\
\hline $\begin{array}{l}\text { Jeune } \\
\text { chercheuse E2 }\end{array}$ & Initiale & U1 & $\begin{array}{c}\text { Sc.de } \\
\text { l'éducation }\end{array}$ & 16 & oui \\
\hline $\begin{array}{l}\text { Jeune } \\
\text { chercheuse E3 }\end{array}$ & Initiale & U1 & $\begin{array}{c}\text { Sc.de } \\
\text { l'éducation }\end{array}$ & abandon & non \\
\hline $\begin{array}{l}\text { Jeune } \\
\text { chercheuse E4 }\end{array}$ & Initiale & $\mathrm{U} 2$ & $\begin{array}{c}\text { Sc.de } \\
\text { l'éducation }\end{array}$ & abandon & non \\
\hline $\begin{array}{l}\text { Jeune } \\
\text { chercheuse E5 }\end{array}$ & Initiale & U3 & Psychologie & 14 & oui \\
\hline $\begin{array}{l}\text { Jeune } \\
\text { chercheuse E6 }\end{array}$ & Initiale & U4 & Sociologie & 16 & non \\
\hline $\begin{array}{l}\text { Jeune } \\
\text { chercheuse E7 }\end{array}$ & Initiale & U4 & Sociologie & abandon & non \\
\hline $\begin{array}{l}\text { Jeune } \\
\text { chercheuse E8 }\end{array}$ & Initiale & U4 & Sociologie & 16 & oui \\
\hline $\begin{array}{l}\text { Jeune } \\
\text { chercheuse E9 }\end{array}$ & Initiale & U4 & Sociologie & 16 & oui \\
\hline $\begin{array}{l}\text { Jeune } \\
\text { chercheuse } \\
\text { E10 }\end{array}$ & Continue & U5 & $\begin{array}{c}\text { Sc.de } \\
\text { l'éducation }\end{array}$ & 16 & ne sait pas \\
\hline $\begin{array}{l}\text { Jeune } \\
\text { chercheuse } \\
\text { E11 }\end{array}$ & Continue & U3 & $\begin{array}{l}\text { Sc.de } \\
\text { l'éducation }\end{array}$ & abandon & non \\
\hline
\end{tabular}

\subsection{Instrumentation}

Nous avons élaboré le guide d'entretien à partir de vingt entretiens exploratoires (Quivy et Van Camphenhoudt, 2006). Les résultats de ces entretiens exploratoires ont permis de dégager une certaine exhaustivité dans les thèmes abordés; ils ont également servi au pilotage du protocole d'entretien. Le guide d'entretien était composé de cinq grandes thématiques: 1) le premier 
contact entre le directeur et la jeune chercheuse; 2) le déroulement des rencontres; 3) la relation interpersonnelle au sein du binôme; 4) l'aide apportée par le directeur dans la construction du travail de recherche de la jeune chercheuse; 5) la perception du rôle de chacun et leurs attentes. Le tableau ci-dessous présente un extrait du guide d'entretien (voir Tableau 2).

\section{Tableau 2}

Extrait du guide d'entretien

\begin{tabular}{|c|c|c|}
\hline Thématique & Questions & Relances \\
\hline $\begin{array}{c}\text { La relation } \\
\text { interpersonnelle }\end{array}$ & $\begin{array}{l}\text { Vous sentez-vous } \\
\text { plutôt proche ou } \\
\text { distant de votre } \\
\text { directeur? }\end{array}$ & $\begin{array}{l}\text { C'est-à-dire? Qu'entendez-vous par proche/distant? } \\
\text { Êtes-vous satisfait de cette proximité/distance? } \\
\text { Pourquoi? Avez-vous des discussions extra-mémoire? } \\
\text { Est-ce que vous tutoyez/vouvoyez votre directeur? } \\
\text { Pour quelles raisons? Est-ce que votre directeur vous } \\
\text { soutient, vous encourage? De quelles manières? Êtes- } \\
\text { vous satisfait de son soutien/encouragement? } \\
\text { Pourquoi? }\end{array}$ \\
\hline
\end{tabular}

\subsection{Déroulement}

Les 11 jeunes chercheuses ont été interviewées sur le terrain en milieu d'année, de manière à ce qu'elles aient fait l'expérience de leur accompagnement et en comprennent suffisamment le fonctionnement pour pouvoir en discuter. Il s'agissait d'entretiens semi-directifs d'une durée moyenne d'une heure. La première question du guide Comment s'est fait le choix du directeur ? a fait office de consigne initiale. À noter qu'en fonction des laboratoires, soit l'étudiant choisit son directeur, soit il lui est imposé. Cette première question du guide portait sur le premier contact entre la jeune chercheuse et son directeur de recherches, de manière à poser un cadre chronologique à l'entretien, depuis le choix de son directeur jusqu'au moment de notre entretien. Tous les entretiens ont été enregistrés et transcrits dans leur intégralité par l'auteure de l'article.

\subsection{Considérations éthiques}

Pour garantir l'anonymat des enquêtées, les noms des sujets ont été remplacés par une lettre de l'alphabet suivie d'un chiffre (par exemple: E1, E2). Il en a été de même pour le nom des universités (par exemple : U1, U2). Par ailleurs, nous avons fait le choix de ne pas mentionner dans les transcriptions les domaines d'étude ou les sujets de recherche évoqués par les enquêtées. Outre ces précautions, il faut signaler une vigilance particulière concernant le discours des jeunes 
chercheuses qui souhaitaient poursuivre leur cursus en doctorat, certains passages trop reconnaissables n'apparaissent pas dans les transcriptions. Enfin, toutes les publications issues de ce travail de recherche ont été envoyées à l'ensemble du groupe d'étude.

\subsection{Méthode d'analyse des résultats}

Nous avons procédé à une analyse thématique par codage émergeant (Mucchielli, 1993) à l'aide du logiciel de traitement de données qualitatives NVivo (Welsh, 2002). Ce logiciel permet de découper les données en vue de l'analyse de contenu. À cette fin, pour l'analyse des entretiens, nous avons défini des catégories. Elles ont été créées à partir de la définition des trois dimensions, couplée à une première lecture des commentaires des jeunes chercheuses. Nous avons constitué notre grille de codage selon le modèle $C$ de L'Écuyer (1990), qui permet une adaptation progressive d'une grille préexistante aux nouvelles données. Une mesure de fidélité interjuge a été réalisée de manière informelle sur un tiers des entretiens.

La grille de codage est composée de trois niveaux de catégorisation: 1) catégories principales, 2) catégories secondaires et 3) sous-catégories (voir Tableau 3). Les catégories principales sont les suivantes : place du collectif, termes employés, choix du sujet, écrits, premier contact, rendez-vous individuels, contacts extérieurs aux rendez-vous individuels, aide apportée par le directeur, relation interpersonnelle, soutenance, satisfaction/insatisfaction, perception du rôle de l'étudiant, perception du rôle du directeur de recherches. Les catégories secondaires correspondent aux trois dimensions, scientifique, institutionnelle et relationnelle, qui elles-mêmes ont été subdivisées en sous catégories.

\section{Tableau 3}

Extraits du codage des entretiens sous Nvivo

\begin{tabular}{|c|c|c|}
\hline $\begin{array}{l}\text { Catégories } \\
\text { principales }\end{array}$ & Catégories secondaires & Sous catégorie \\
\hline \multirow{3}{*}{$\begin{array}{l}\text { Aide apportée par } \\
\text { le directeur }\end{array}$} & Dimension scientifique & $\begin{array}{l}\text { Aide théorique } \\
\text { Aide méthodologique } \\
\text { Aide scripturale }\end{array}$ \\
\hline & Dimension relationnelle & Soutien psychologique \\
\hline & Dimension institutionnelle & Aide organisationnelle \\
\hline
\end{tabular}




\section{Résultats}

Pour cet article, nous avons choisi de présenter uniquement ce que l'on observe de façon commune et récurrente dans l'ensemble des discours des jeunes chercheuses et qui semble constituer une spécificité des binômes directeur homme - jeune chercheuse. Ces éléments du discours se sont retrouvés de manière transversale dans chacune des thématiques abordées lors des entretiens (voir tableau 4).

\section{Tableau 4}

Analyse des entretiens

\begin{tabular}{|c|c|c|}
\hline Dimensions & Récurrences dans les discours & $\begin{array}{l}\text { Nombre de jeunes } \\
\text { chercheuses } \\
\text { concernées }\end{array}$ \\
\hline \multirow[t]{2}{*}{$\begin{array}{l}\text { Dimension } \\
\text { scientifique }\end{array}$} & $\begin{array}{l}\text { 1) Dévalorisation de soi en tant que jeune } \\
\text { chercheuse }\end{array}$ & 4 \\
\hline & $\begin{array}{l}\text { 2) Utilisation d'un vocabulaire très } \\
\text { hiérarchique et scolaire, opposé au } \\
\text { vocabulaire de la relation } \\
\text { d'accompagnement de recherches }\end{array}$ & 11 \\
\hline \multirow{4}{*}{$\begin{array}{l}\text { Dimension } \\
\text { institutionnelle } \\
\text { Dimension } \\
\text { relationnelle }\end{array}$} & $\begin{array}{l}\text { 3) Difficulté de prise de contact et de } \\
\text { rencontres seule à seul }\end{array}$ & 7 \\
\hline & 4) Usage systématique du vouvoiement & 11 \\
\hline & $\begin{array}{l}\text { 5) Perception du directeur comme une } \\
\text { figure d'autorité }\end{array}$ & 5 \\
\hline & $\begin{array}{l}\text { 6) Perception de la relation comme } \\
\text { ambiguë }\end{array}$ & 6 \\
\hline
\end{tabular}

\subsection{Dimension scientifique}

4.1.1 Dévalorisation de soi en tant que jeune chercheuse

Sur les 11 interrogées, 4 jeunes chercheuses abordent dans leur discours des éléments qui portent sur la dévalorisation de soi en tant que jeune chercheuse. E6 et E7 évoquent un complexe d'infériorité : J'ai toujours aussi un peu ce complexe d'infériorité vis-à-vis d'un prof (E6), Je ressortais toujours autant angoissée et complexée de mon ignorance à l'issue des entretiens (E7). E1 et E7 éprouvent de la honte à faire part de leurs difficultés à leur directeur : J'ai peur qu'il prenne ça pour de la flemme (E1), J'ai un peu honte de lui [...] montrer que je suis paumée (E7), J'avais peur [...] de mal paraître (E1). Dévoiler ses difficultés est perçu comme un signe de faiblesse ou d'incompétence, et non comme faisant partie intégrante du processus 
d'apprentissage. E2, quant à elle, se sent surestimée par son directeur et ne se sent donc pas à la hauteur de ses attentes: C'est ça mon problème, c'est qu'il me surestime (E2). On perçoit ici des éléments liés au syndrome de l'imposteur, que l'on retrouve également dans les discours de E1 et de E7. Ces jeunes chercheuses préfèrent fuir les rencontres et les contacts avec leur directeur plutôt que d'être démasquées comme incompétentes : Je bloque, je manque d'oser y aller [...] [j'ai peur] de ne pas dire ce qu'il faut ou d'apparaître complètement à côté de la plaque [...] et sincèrement je n'ose pas (E1), Je suis vite amenée à paniquer dans les conversations parce que j'ai peur que le voile tombe, qu'il se rende compte que je rame, je rame [...] j'avais peur qu'il se rende compte que je faisais semblant d'être une bonne élève (E7).

4.1.2 Utilisation d'un vocabulaire très hiérarchique et scolaire, par opposition au vocabulaire de la relation d'accompagnement de recherches

Le directeur de recherches semble être perçu par les jeunes chercheuses comme une autorité hiérarchique, à l'instar de ce qu'elles ont pu expérimenter ou ressentir en licence. Elles le perçoivent davantage comme un enseignant dont l'autorité est difficilement discutable, et non comme un accompagnateur avec qui la recherche se co-construit. D'ailleurs, les 11 jeunes chercheuses interrogées utilisent un vocabulaire très scolaire pour décrire leur relation d'accompagnement. Par exemple, E1 utilise le terme de tuteur pour désigner son directeur, mes cours de fac pour nommer les séminaires méthodologiques, de sanction pour évoquer les rétroactions de son directeur et aussi d'évaluation pour désigner l'écrit obligatoire, alors que cet écrit possède aussi une visée formative qu'elle ne semble pas envisager. Les autres jeunes chercheuses utilisent également des termes très scolaires tout au long de leur discours: correction, sanction pour renvoyer aux annotations, professeur, prof pour désigner leur directeur et élève pour se désigner Je suis l'élève, moi (E2). E6 va même jusqu'à parler d'une relation dominant-dominé, et E3 décrit sa posture comme celle d'une jeune chercheuse passive et attentiste (j'attends qu'il m'apprenne), une posture plus proche de celle d'un étudiant que d'une jeune chercheuse. Les termes employés montrent que les jeunes chercheuses se situent encore dans une relation hiérarchique d'enseignant-enseigné, dans la continuité de la licence, davantage que dans une relation d'accompagnement: Il faut que j'accomplisse mon devoir d'élève de master 2 (E8). Dans le prolongement de cette étude, il serait pertinent de comparer le vocabulaire utilisé par les jeunes chercheurs appartenant aux autres binômes : cette observation est-elle 
spécifique à notre groupe d'étude ou est-elle spécifique aux jeunes chercheurs inscrits en master 2?

\subsection{Dimension institutionnelle : difficultés de prise de contact et de rencontres seule à seul}

Elle concerne les difficultés de prise de contact et de rencontres seule à seul. Lors de notre entretien, au mois d'avril, 7 jeunes chercheuses (E1, E2, E4, E5, E6, E7, E10) ont rencontré une seule fois ou aucune fois leur directeur en rendez-vous individuel. Par manque de disponibilité de leur directeur, E2 et E7 l'ont rencontré moins d'un quart d'heure et de manière informelle, E4 ne souhaite plus solliciter de rendez-vous auprès de son directeur par manque d'affinité (Je le sollicite sans le solliciter dans le sens [où] je ne me sens d'aucune affinité avec lui), E10 n'a bénéficié que des rencontres collectives, et E1 appréhende les rencontres en face à face. E1 n'a rencontré son directeur qu'une seule fois, sur sollicitation de ce dernier, et l'entretien s'est déroulé à la cafétéria, un lieu moins institutionnel et plus peuplé que le bureau du directeur. Comme pour E10, les seules autres rencontres ont eu lieu de manière groupale, lors des séminaires méthodologiques. E1 semble rencontrer de réelles difficultés à instaurer une relation et un dialogue avec son directeur: Je lui ai écrit plein de mails que je n'ai jamais réussi à lui envoyer (E1); j'aime bien le système des séminaires [...] sans forcément passer par le rendezvous individualisé où tu te sens assez obligée d'avoir de quoi alimenter la conversation pendant une heure (E1). Nous pouvons remarquer ici que E1 tend à compenser l'absence de relation duale et ses difficultés de communication avec son directeur en tirant profit des interactions entre pairs et des discussions qui ont lieu lors des séminaires méthodologiques : Les séminaires [...] moi je trouve que c'est une forme qui est intéressante [...] c'est intéressant aussi de présenter la difficulté que l'on rencontre pour que tout le monde réagisse (E1). Elle se sert également des séminaires pour se fixer des échéances de travail, ce qui lui permet d'avancer dans sa recherche : Je revois mon tuteur le 8 avril en séminaire; donc, pour le séminaire, j'aurai mes entretiens, [...] je continue à avancer un peu sur mon boulot (E1). E8, quant à elle, a adopté une autre stratégie, elle compense le manque de contact avec son directeur en découvrant ses attentes à travers l'ouvrage méthodologique dont il est l'auteur: Comme il a fait un bouquin de méthodo assez précis et que le cours de méthodo a été fait par lui, c'est vrai qu'on a un peu tous les outils dans ce bouquin qui peuvent nous aider pour le mémoire.

Signalons que 3 jeunes chercheuses $(E 3, E 8, E 11)$ ont rencontré deux ou trois fois leur 
directeur de recherche. Pour E3, les rendez-vous ont été fixés par son directeur, alors que E8 et E11 les ont elles-mêmes sollicités.

Une jeune chercheuse, E9, rencontre son directeur de recherches à l'excès, presque chaque semaine lors de sa permanence. La relation est ambiguë parce que la limite entre la dimension scientifique et la vie privée a été franchie par la jeune chercheuse. En effet, cette dernière aborde des éléments de sa vie privée qui n’ont pas nécessairement de lien avec l'avancement de son travail de recherche : Je lui raconte mes histoires de cul. L'autre jour, je lui dis : je suis en train de draguer un mec, un timide à caractère asocial (E9). Leurs rencontres ont lieu dans des lieux institutionnels, tel que le bureau du directeur; dans des lieux informels, comme les bars ou les restaurants, et dans des cadres domestiques (au domicile du directeur).

\subsection{Dimension relationnelle}

\subsubsection{Usage systématique du vouvoiement}

Aucune jeune chercheuse ne tutoie son directeur de recherche. Les jeunes chercheuses remarquent d'ailleurs une différence avec les binômes directeur-étudiant: Je le vouvoie, il me vouvoie; je sais qu'avec les hommes, ils se tutoient (E11). Je lui ai dit ouais, c'est dégueulasse (sic) : tous les hommes, ils vous tutoient et toutes les femmes, elles vous vouvoient (E9). Si leur directeur leur proposait le tutoiement, elles disent cependant préférer rester sur le mode du vouvoiement. Le principal argument avancé est le respect de la relation hiérarchique : Je suis l'élève, [...] c'est un prof(E2), un prof c'est un prof et l'élève c'est l'élève (E6). Je suis incapable de tutoyer un professeur (E7). Ça reste mon professeur [...], ça reste dans un cadre universitaire (E8).

\subsubsection{Perception du directeur comme une figure d'autorité}

Le directeur est perçu par 5 jeunes chercheuses (E2, E3, E5, E6, E7) comme une figure d'autorité. Dans certains cas, l'autorité peut être destructrice, comme le relate E5: [Mon directeur de MI], c'était quelqu'un d'assez cassant [...], ça me paralysait [...]. Je me sentais infériorisée [...]. Il m'a dit, par exemple, que j'écrivais très mal le français, et que même quelqu'un qui n'était pas francophone aurait beaucoup mieux écrit que moi [...]. Il a dit ça en public (E5). La jeune chercheuse E6 est en co-direction, elle perçoit son directeur comme plus impressionnant et plus autoritaire que sa co-directrice. Elle utilise des termes et des expressions 
tels qu'impressionnée, c'est quelqu'un d'assez important, pas très content, tapé du poing sur la table qui renvoient aux stéréotypes de l'autorité paternelle : d'un point de vue relationnel, déjà c'est une femme, c'est un homme, donc ce n'est pas pareil. Je crois que c'est clair, ce n'est pas du tout pareil [...]. Je suis impressionnée par monsieur $Y[\ldots]$ monsieur Y a un peu tapé du poing sur la table parce que je n'ai pas vu quelque chose dans mon mémoire qui avait de l'importance, [...] Elle s'occupe beaucoup de l'orthographe, mais c'est bien parce que si monsieur Y voyait les fautes d'orthographe, il ne serait pas très content. La manière dont elle parle de sa directrice renvoie aux stéréotypes de la femme secrétaire. Elle s'occupe beaucoup de l'orthographe et de l'image maternelle. Elle la perçoit plus disponible, plus accessible, et davantage dans le relationnel. La jeune chercheuse utilise des expressions telles que: Elle s'occupe, elle est humaine, elle prend en compte, elle est plus terre-à-terre, elle prend plus en compte ce qu'on veut faire et ce qu'on est. Elle a une proximité relationnelle avec sa directrice qu'elle ne semble pas avoir avec son directeur: Je ne regarde pas monsieur $Y$ de la même manière que madame $X$ : monsieur $Y$, c'est plus de l'admiration; madame $X[\ldots]$ elle fait plus accessible. Son directeur est perçu comme inaccessible, mais intellectuellement brillant, sous-entendant que sa directrice serait plus accessible, mais moins brillante. Nous retrouvons cette admiration intellectuelle dans le discours de E2 : Il est super intéressant, il a une culture impressionnante; dans celui de E7: C'est quelqu'un d'extrêmement charismatique et de E3 : Il est hyper compétent. D'après le discours de E3, E5 et E6, leur perception de l'accompagnement de recherche serait davantage en adéquation avec la perception que s'en feraient les directrices, à savoir l'importance du relationnel, de l'individu. Nous venons de le voir dans le discours de E6, nous le retrouvons dans le discours de E5. Cette jeune chercheuse compare sa relation avec son directeur en master 1 et sa co-directrice en master 2: L'année dernière, on parlait que du travail [...]; cette année, je peux dire que je suis de mauvaise humeur (E5). Les insatisfactions relationnelles exprimées par E3 vont aussi dans ce sens : [ce que]j'attendrais de lui, ce serait de prendre plus de mes nouvelles, en me disant ben là tu en es où, tu as besoin d'aide. La jeune chercheuse s'attend à ce que son directeur prenne l'initiative des rencontres et sur des aspects d'ordre relationnel (prendre de ses nouvelles). Ainsi, la jeune chercheuse tend à interpréter l'absence de sollicitation de son directeur d'un point de vue du relationnel, de l'affect (un manque d'intérêt envers elle), plutôt que d'un point de vue scientifique (c'est le rôle du jeune chercheur de solliciter son directeur). 


\subsubsection{Perception de la relation comme ambiguë}

Dans leur discours, 6 jeunes chercheuses (E1, E3, E4, E5, E7, E9) font état d'une ambiguïté dans leur relation. E9 semble percevoir sa relation d'accompagnement comme une relation amicale : On est devenu officiellement amis, voire comme une relation amoureuse : Mon directeur, c'est un mec que j'aime bien draguer parce que, comme il ne répond pas, c'est encore plus excitant. La jeune chercheuse E5 compare ses deux relations: Je me sens beaucoup mieux avec une directrice de mémoire qu'avec un directeur de mémoire [...], c'est-à-dire, c'est beaucoup plus clair d'avoir affaire à une femme plutôt qu'à un homme [...] : Je me pose moins de questions (E5). Les jeunes chercheuses E1 et E3 sont aussi dans une relation qu'elles jugent inconfortable et tendent, de fait, à fuir les rencontres duales avec leur directeur. Cette ambiguïté relationnelle se retrouve dans le discours de E7. Elle parle de son directeur en utilisant des termes qui rappellent la relation de couple: Si je vais le voir et que je lui dis ça ne va pas, il va m'écouter, mais il ne va pas m'entendre (E7). J'attendais qu'il me fasse ressentir qu'il pense à mon mémoire en dehors de nos entrevues (E7). La jeune chercheuse a l'impression de ne pas être comprise par son directeur, et elle l'interprète comme un manque d'attention à son égard. Il en est de même dans l'entretien de E4. Selon elle, son directeur ne serait pas intéressé par son sujet de mémoire. Elle envie beaucoup la relation qu'il entretient avec une autre jeune chercheuse qui travaille sur ses problématiques. La manière dont elle parle de cette situation fait penser à une relation empreinte de tromperie et d'infidélité : Lui, il pense à elle, mais bon, j'en ai pris mon parti, je travaille avec d'autres chercheurs (E4). J'espérais tomber sur un directeur de recherche avec une vraie relation (E4). J'aurais voulu qu'il soit plus proche (E4). E3 utilise également ce même champ lexical : Je ne lui pose pas la question de savoir si je lui plais ou si je ne lui plais pas (E3).

\section{Discussion}

\subsection{Dimension scientifique : une posture d'étudiante}

Au niveau de la dimension scientifique, la recherche de Lafay et al. (2003) avait mis en exergue la sensation des jeunes chercheuses de ne pas être prises au sérieux par leur directeur de recherches (Lafay et al., 2003). Dans notre étude, elles ne se sentent pas directement jugées sur leurs compétences, mais elles se jugent elles-mêmes en imaginant le jugement de leur directeur.

Notre recherche fait apparaitre d'autres résultats. Les jeunes chercheuses semblent ne pas avoir perçu l'évolution des rôles de chacun et de la relation pédagogique depuis leur entrée en 
master. Elles tendent à rester dans une posture d'étudiante, à considérer leur directeur comme un enseignant, et leur relation pédagogique comme une relation enseignant-enseigné, dans la continuité de la licence.

Les jeunes chercheuses interrogées montrent en effet des difficultés à utiliser le vocable du jeune chercheur. Par exemple, certains termes utilisés laissent penser que les jeunes chercheuses perçoivent davantage les rétroactions de leur directeur comme une sanction, telle qu'elles ont pu le vivre en licence, que comme une aide à l'apprentissage nécessaire au développement de la compétence rédactionnelle. Le vocabulaire utilisé est très scolaire, et les marqueurs verbaux utilisés témoignent de la persistance du rapport hiérarchique. Ce qui nous interpelle ici n'est pas tant l'usage du vouvoiement que les justifications qu'elles donnent à cet usage. Elles se placent dans une posture d'étudiante et considèrent leur relation duale d'accompagnement dans la lignée de leur relation pédagogique groupale de licence, c'est-à-dire dans une relation très hiérarchique et hiérarchisée. Elles semblent vouloir rester dans une relation groupale enseignant-enseignés qu'elles connaissent et qui les rassure, plutôt que d'entrer dans une relation jeune chercheuse-directeur de recherches qui est attendue à ce niveau universitaire, mais qui leur est inconnue. Cette manière de penser la relation peut avoir des effets sur la qualité de la formation. Si la jeune chercheuse ne remet pas en cause la parole de son directeur, le dialogue critique risque de ne pas contribuer à l'évolution de sa réflexion, parce que le conflit sociocognitif n'aura pas lieu (Montgomery, Morin, et Demers, 2010).

\subsection{Dimension institutionnelle : une communication difficile}

Au niveau de la dimension institutionnelle, Powles (1989) ainsi que Schmidt et Umans (2014) mentionnaient que les jeunes chercheuses passaient moins de temps avec leur directeur et que le contact était moins fréquent qu'avec leurs homologues masculins. Les résultats de notre recherche vont dans ce sens : la plupart des jeunes chercheuses de notre groupe d'étude ont éprouvé des difficultés à contacter leur directeur de recherches, à prendre un rendez-vous pour le rencontrer ou à le rencontrer seule à seul. Elles ont tendance à reproduire le cadre communicationnel qu'elles ont pu vivre en licence, c'est-à-dire des contacts réduits avec l'enseignant en dehors d'une situation de classe ou des rencontres exclusivement groupales. Ainsi, les jeunes chercheuses semblent ne pas avoir perçu l'évolution communicationnelle de la licence au master, ou alors elles ne semblent pas encore parvenir à adopter les nouveaux codes 
qui sont attendus à ce niveau universitaire, comme ne pas attendre les sollicitations de la part de son directeur (Gerard, 2009).

Cette difficulté de prise de contact n'est pas sans conséquence, car elles se privent d'une aide dans leur formation à la recherche par la recherche. Face à cette autoprivation, deux comportements opposés se distinguent: 4 jeunes chercheuses sont restées avec leurs insatisfactions et leurs difficultés et ont choisi d'abandonner leur mémoire; les 7 autres jeunes chercheuses ont développé des stratégies de compensation. Par exemple, certaines d'entre elles ont compensé l'absence de rencontres en assistant à des séminaires de recherche et en tirant profit. Une autre se forme à la recherche en prenant appui sur l'ouvrage écrit par son directeur, une autre encore travaille et se fait relire par ses pairs.

\subsection{Dimension relationnelle : l'importance de la relation interpersonnelle}

Dans leur passage de la licence au master, les jeunes chercheuses semblent éprouver des difficultés dans la gestion de la relation interpersonnelle duale d'accompagnement.

$\mathrm{Au}$ niveau de la dimension relationnelle, les recherches montraient que les jeunes chercheuses étaient en attente d'une certaine proximité relationnelle (Gerard, 2009; Hammick et Acker, 1998; Juniper et al., 2012). Les résultats de notre recherche vont dans ce sens. Pour les jeunes chercheuses de notre groupe d'étude, les éléments qui ont trait à la dimension relationnelle constituent le point central de la relation d'accompagnement. Les jeunes chercheuses sont avant tout en recherche d'une humanité et d'une proximité relationnelle qu'elles ne retrouvent pas ou peu chez leur directeur. Elles sont en demande d'un soutien psychologique et de discussions extra mémoire. De la même manière que les directrices de recherches, elles tendent à envisager l'individu dans son ensemble, et non pas uniquement sous un angle scientifique et professionnel. Si le directeur n'explicite pas sa perception de la relation d'accompagnement et du rôle de chacun, la jeune chercheuse risque d'interpréter cette distance relationnelle comme un abandon et un désintérêt vis-à-vis de son sujet, voire un rejet de sa personne, et non comme le choix d'une posture d'accompagnement. Cette perception négative peut conduire à l'abandon (Gremmo et Gerard, 2008) parce que les insatisfactions liées à la dimension relationnelle semblent avoir un impact négatif sur les deux autres dimensions : les jeunes chercheuses évitent les contacts ou les rencontres avec leur directeur (dimension institutionnelle), se privant ainsi d'une aide pour l'avancement de leur recherche et le développement de leurs compétences de chercheur 
(dimension scientifique).

Les résultats de recherche précédemment cités, font également état de préjugés machistes et de harcèlement sexuel. Dans notre groupe d'étude, ces éléments ne sont pas apparus. Néanmoins, une jeune chercheuse de notre échantillon avec laquelle nous avons gardé le contact, nous a fait part d'un harcèlement sexuel de la part de son directeur pendant son doctorat. Nous pensons que la relation d'accompagnement de master est peut-être trop courte pour observer ce genre de dérives.

\section{Conclusion}

Notre recherche est partie des deux constats suivants: 1) À l'entrée en master, l'étudiant expérimente pour la première fois une relation pédagogique duale d'accompagnement; 2) en master, les jeunes chercheuses sont plus souvent accompagnées dans leur recherche par un directeur du sexe opposé que les jeunes chercheurs. Notre question de recherche était la suivante : Comment les jeunes chercheuses accompagnées dans leur recherche par un directeur homme perçoivent-elles cette relation pédagogique à deux? Autrement dit, quelles perceptions ont-elles d'elles-mêmes et de leur directeur de recherches, et ce, du point de vue des trois dimensions (scientifique, institutionnelle et relationnelle)? Pour mener cette recherche, nous avons interrogé onze jeunes chercheuses inscrites en master 2. Les résultats montrent que les jeunes chercheuses perçoivent leur rôle, le rôle de leur directeur et leur relation d'accompagnement de manière très scolaire et hiérarchisée, comme une relation pédagogique enseignant-enseigné, comme elles ont pu l'expérimenter en licence (dimension scientifique). Elles éprouvent, par ailleurs, des difficultés à communiquer avec leur directeur de recherches (dimension institutionnelle) et à gérer la relation interpersonnelle (dimension relationnelle). Enfin, la dimension relationnelle est considérée par les jeunes chercheuses comme le point central de la relation d'accompagnement et constitue leur principale source d'insatisfaction, ce qui a un impact négativement sur les deux autres dimensions.

Notre étude présente néanmoins des limites. Notre échantillon de jeunes chercheuses est trop limité pour généraliser les résultats à l'ensemble de ces binômes; une étude similaire pourrait être menée sur un échantillon plus grand. Par ailleurs, il serait nécessaire d'effectuer des comparaisons avec des participants appartenant aux autres binômes (directeur - jeune chercheur, directrice - jeune chercheur, directrice - jeune chercheuse), de manière à vérifier si le vécu des 
participants serait différent ou identique à celui de notre échantillon. Par ailleurs, le point de vue des directeurs de recherche serait nécessaire pour obtenir leurs perceptions concernant leur relation d'accompagnement avec une jeune chercheuse. Enfin, il serait pertient d'examiner la relation pédagogique ailleurs qu'en France, par exemple dans un pays anglo-saxon, afin de voir si la perception de la relation serait différente.

Néanmoins, en ce qui concerne ces binômes à risque, nous conseillons aux directeurs de recherche de commencer par expliciter leur rôle, leurs attentes, ainsi que les limites de leur accompagnement, de manière à ce que la jeune chercheuse parvienne à mieux appréhender le fonctionnement de cette nouvelle relation pédagogique. Il peut être opportun de fixer avec elle les dates des rencontres pour éviter les difficultés de sollicitation, et de leur demander des écrits réguliers pour les inciter à faire part de leurs difficultés. Enfin, en instaurant une relation de confiance, en donnant des appréciations positives sur le travail effectué, le directeur permettrait peut-être à la jeune chercheuse de développer davantage sa confiance en elle. Quoi qu'il en soit, cette étude permet d'apporter des éléments de réflexion et de compréhension à propos de cette relation d'accompagnement.

\section{ENGLISH TITLE - Females enrolled in master degree programs supervised by male supervisors: a perception of their supervision}

SUMMARY - We started from two observations: 1) when students enter the master's degree, they experience for the first time a new pedagogical relationship, which is supervision; 2) in the master's degree, young female researchers are most often supervised by a male supervisor. How do young female researchers supervised by a male supervisor perceive the supervision? What perceptions do they have of themselves and their supervisor, in terms of the three dimensions: scientific, institutional and relational? To conduct this research, we interviewed eleven female students enrolled in a master degree program. The results show that young researchers perceive their role, the role of their supervisor and their supervision relationship in a very hierarchical relation. They also experience difficulties in communicating with their supervisors and managing the interpersonal relationships. Finally, the relational dimension is considered by female students as the central point of their supervision relationship and their main source of dissatisfaction.

KEY WORDS - Supervision, master, supervisor, cross-sex, female. 


\section{TíTUlo - Las investigadoras en formación bajo la dirección de un investigador de sexo masculino: percepción de la relación de acompañamiento.}

RESUMEN - Nuestra investigación parte de dos hechos: 1) al comienzo de los estudios de máster, el estudiante experimenta por primera vez un nuevo tipo de relación pedagógica, dual: la relación de acompañamiento para realizar la memoria; 2) a nivel de máster, las estudiantes en formación a la investigación son dirigidas por un director de sexo opuesto con más frecuencia que los estudiantes. Nuestra investigación plantea las preguntas siguientes: ¿cómo perciben esta relación pedagógica las estudiantes en formación a la investigación dirigidas por un director de sexo masculino? ¿Qué percepción tienen estas estudiantes de sí mismas y de su director, sobre tres dimensiones: científica, institucional y relacional? Para desarrollar esta investigación, se hicieron entrevistas con once estudiantes en formación a la investigación de sexo femenino, inscritas en segundo año de máster. Los resultados muestran que estas estudiantes perciben su papel, el papel de su director y su relación de acompañamiento de forma muy escolar y jerarquizada. Además, experimentan dificultades para comunicar con su director de investigación (dimensión institucional) y para manejar la relación interpersonal (dimensión relacional). Finalmente, la dimensión relacional es considerada por estas estudiantes como el punto central de la relación de acompañamiento y constituye su principal fuente de insatisfacción.

Palabras Clave - Supervisión, dirección de investigación, acompañamiento, género, estudiante de sexo femenino.

\section{Références}

Bandura, A. (2003). Auto-efficacité. Le sentiment d'efficacité personnelle. Bruxelles, Belgique : De Boeck.

Boyd, S. and Wylie, C. (1994). Workload and stress in New Zeland universities. Wellington, New Zealand: New Zealand council for educational research and the association of university staff of New Zeland.

Carré, P. (2005). L’apprenance: Vers un nouveau rapport au savoir. Paris, France: Dunod Éditeur.

Collectif de lutte antisexiste contre le harcèlement sexuel dans l'enseignement supérieur (2014). Le harcèlement sexuel dans l'enseignement supérieur et la recherche. Paris, France : Collectif de lutte antisexiste contre le harcélement sexuel dans l'enseignement supérieur.

Conrad, L. (1994). Gender and postgraduate supervision. In O. Zuber-Skerritt and Y. Ryan (eds), Quality in postgraduate education (p. 51-58). London, United Kingdom: Kogan Page.

Dahan, A. (2007). Supervision and schizophrenia: the professional identity of Ph.D supervisors and the mission of students' professionalisation. European journal of education, 42(3), 335349. 
Gerard, L. (2009). L'accompagnement en contexte de formation universitaire : étude de la direction de mémoire comme facteur de réussite en Master. Université de Lorraine, Nancy. récupéré du site de la revue : http://tel.archives-ouvertes.fr/tel-00498380/.

Gerard, L. (2014). Le doctorat: un rite de passage. Analyse du parcours doctoral et post doctoral. Paris, France : Tétraèdre.

Germain, M. et Gremillet, M. (2000). La guidance de mémoire : comment diriger et élaborer un mémoire universitaire. Paris, France : L'Harmattan.

Gillespie, N. A., Walsh, M., Winefield, A. H., Dua, J. and Stough, C. (2001). Occupational stress in universities: staff perceptions of the causes, consequences and moderators of stress. Work and stress, 15(1), 53-72.

Gremmo, M.-J. et Gerard, L. (2008). Accompagner les apprentis chercheurs : jeux et enjeux de la direction de mémoire. Recherche et formation, 59, 43-58.

Hammick, M. and Acker, S. (1998). Undergraduate research supervision: a gender analysis. Studies in higher education, 23(3), 335-347.

Heinrich, K. T. (1991). Loving partnership, dealing with sexual attraction and power in doctoral advisement relationship. Journal of higher education, 62(5), 514-538.

Herzlich, C. (2002). Réussir sa thèse en sciences sociales. Paris, France : Nathan éditeur.

Juniper, B., Walsh, E., Richardson, A. and Morley, B. (2012). A new approach to evaluating the well-being of $\mathrm{PhD}$ research students. Assessment and evaluation in higher education, 37(5), 563-576.

Karpenko, V. and Gidycz, C. A. (2012). The supervisory relationship and the process of evaluation: recommendations for supervisors. The clinical supervisor, 31(2), 138-158.

Knowles, M. (1975). Self-directed learning: a guide for learners and teachers. New York, New York: Association Press.

Lafay, N., Manzanera, C., Papet, N., Marcelli, D. et Senon, J. L. (2003). Les états dépressifs de la post-adolescnce. Résultats d'une enquête menée chez 1521 étudiants de l'université de Poitiers. Annales Médico-Psychologiques, 161, 147-153.

Langevin, L. et Bruneau, M. (2000). Enseignement supérieur. Vers un nouveau scénario. Issyles-Moulineaux, France : ESF éditeur. 
Le Bouëdec, G. et de La Garanderie, A. (1993). Les études doctorales en sciences de l'éducation : pour un accompagnement personnalisé des mémoires et de thèses. Paris, France : L'Harmattan.

L'Écuyer, R. (1990). Méthodologie de l'analyse développementale de contenu. Méthode GPS et Concept de Soi. Québec, Québec : Presses de l'Université du Québec.

Leduc, A. (1990). La direction des mémoires et des thèses. Brossard, Québec : Behaviora.

Lee, A. and Boud, D. (2009). Framing doctoral education as pratice. In D. Boud and A. Lee (eds), Changing practices of doctoral education (p. 10-25). Oxon, New York: Routledge.

Montgomery, C., Morin, Y. et Demers, S. (2010). Le stress, les stratégies d'adaptation, le locus de contrôle et l'épuisement professionnel chez les professeurs universitaires francophones. Revue Canadienne d'enseignement supérieur, 40(1), 69-99.

Mucchielli, R. (1993). Le questionnaire dans l'enquête psycho-sociale. Connaissance du problème, applications pratiques. Paris, France : ESF éditeur.

Over, R., Over, J., Meuwissen, I. and Lancaster, S. (1990). Publication by men and women with same-sex and cross-sex PhD supervision. Higher education, 20, 381-391.

Paul, M. (2004). L'accompagnement: une posture professionnelle spécifique. Paris, France: L'Harmattan.

Phillips, E. and Pugh, D. (1994). How to get a PhD. London, United Kingdom: Allen and Unwin. Powles, M. (1989). How's the thesis going? Former postgraduates' and their supervisors' views on lengthy candidature and dropout. Melbourne, Australia: University of Melbourne.

Quivy, R. et Van Camphenhoudt, L. (2006). Manuel de recherche en sciences sociales. Paris, France : Dunod Éditeur.

Royer, C. (1998). Vers un modèle de direction de recherche doctorale en sciences humaines. Sainte-Foy, Québec : Presses de l'Université du Québec.

Schmidt, M. and Umans, T. (2014). Experiences of well-being among female doctoral students en Sweden. International journal of qualitative studies on health and well being, 9, 1-13.

Seagram, B. C., Gould, J. and Pyke, S. W. (1998). An investigation of gender and other variables on time to completion of doctoral degrees. Research in higher education, 39(3), 319-335.

Welsh, E. (2002). Dealing with data: using Nvivo in the qualitative data analysis process. Forum: Qualitative Social Research-FQS, 3(2). 
Madame Laëtitia Gerard est docteure en Sciences de l'éducation, consultante internationale et chercheure associée au laboratoire EXPERICE de l'Université Paris 13.

\section{Correspondance}

gerard.laetitia@gmail.com

\section{Contribution de l'auteure}

Laëtitia Gerard : $100 \%$

Ce texte a été révisé par Karina Da Rocha

Texte reçu le : 19 mars 2016

Version finale reçue le : 2 mai 2017

Accepté le : 17 mai 2017 